\title{
Research Paper \\ Relationship Between Basic Psychological Needs and Mental Vitality in the Elderly
}

\author{
* Mehdi Arabzadeh ${ }^{1}$
}

1. Department of Educational Psychology, Faculty of Psychology and Education, Kharazmi University, Tehran, Iran.

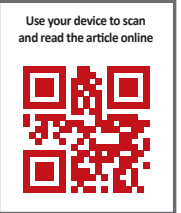

Citation: Arabzadeh M. [Relationship Between Basic Psychological Needs and Mental Vitality in the Elderly (Persian)]. Iranian Journal of Ageing. 2017; 12(2):170-179. http://dx.doi.org/10.21859/sija-1202170

http://dx.doi.org/10.21859/sija-1202170

Received: 02 Jan. 2017

Accepted: 11 Apr. 2017

Keywords:

Mental vitality,

Basic psychological

needs, Elderly

\section{A B STRACT}

Objectives Subjective vitality refers to a positive feeling of aliveness and energy. This condition is important in the aging period; therefore, the purpose of this study was to investigate the relationship between basic psychological needs with subjective vitality in the elderly.

Methods \& Materials It is a functional and cross-sectional study. The research sample included 235 elderly who were selected by convenience sampling method. The participants completed the subjective vitality and basic psychological needs scales. To conduct the necessary descriptive and inferential statistical operations, SPSS (Version 23) was used. Mean and standard deviation were utilized to analyze the data in the descriptive statistics section, and in inferential statistics section, step-by-step multiple regressions were used to predict subjective vitality.

Results The results showed that subjective vitality has a positive and meaningful relationship with autonomy $(P<0.01, r=0.60)$, competence $(P<0.01, r=0.53)$, and relatedness $(P<0.01, r=0.58)$ in the elderly population. Also, the basic psychological needs can be explained by $31 \%$ of variance subjective vitality in elderly people.

Conclusion The basic psychological needs are considered as an important factor for maintaining subjective vitality in the elderly. Therefore, understanding the basic psychological needs and strengthening these factors can be an effective method for enhancing the subjective vitality of elderly people.

\section{Extended Abstract}

\section{Objectives}

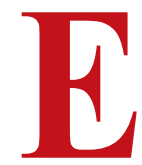

lderliness is a sensitive period in human life in which individuals are exposed to potential threats, such as increased chronic illness, loneliness and isolation, and lack of social support. Due to their physical and mental incapability, their individual independence is threatened in many cases. Therefore, the present study aims at addressing the issues related to sensitivity and vulnerability of the elderly group. The study also addresses the important issue of mental vitality and fulfilling their basic psychological needs. Accordingly, the purpose of this study is to anticipate and explain mental vitality of the elderly based on their basic psychological needs. In other words, is the study aimed to answer the basic question: which of the essential psychological needs plays a greater role in anticipating mental vitality in the elderly? The response to this question is of great help for those who are active in the field of aging-related studies.

\section{Methods \& Materials}

The method of the present study is descriptive of the correlation type and applicable in terms of its purpose. The statistical population of this study included all the

\section{* Corresponding Author:}

Mehdi Arabzadeh, PhD

Address: Department of Educational Psychology, Faculty of Psychology and Education, Kharazmi University, Tehran, Iran.

Tel: +98 (912) 0475660

E-mail: mehdi_arabzadeh@hotmail.com 
elderly men and women in the city of Tehran. From this population, using the Cochran's formula, a sample of 235 people including 100 women and 135 men was selected. For data analysis, the inferential statistics (Pearson correlation coefficient and stepwise multiple regression) were used, and for analyzing the information, the software SPSS version 23 was employed. The present research was approved on April 11, 2016 by the Ethics Committee of the Department of Educational Psychology of the Faculty of Psychology and Educational Sciences of Kharazmi University. The tools used in this research for collecting data were as follows:

\section{Mental Vitality Scales}

This scale was developed by Ryan and Frederick based on the self determination theory of Deci and Ryan [2]. The internal consistency of this scale in their research was reported to be $96 \%$ [5]. This scale has 7 questions, which are scored on a 7-degree Likert scale. Scores range from 7 to 35 . Based on Cronbach's alpha, the reliability of this tool in the present study was equal to 0.79 .

\section{Basic Psychological Needs Scale}

The Deci and Ryan Scale was used [3]. This scale is comprised of 21 questions of which 7 questions assess the psychological need of self management, 6 questions assess the need of competence, and 8 questions assess the need for communication. The questions are based on the 5-degree Likert scale from totally true to totally untrue. The reliability of the said questionnaire has been reported as equal to 0.90 [4]. In the present study, the reliability of this scale by using the Cronbach's Alpha method was obtained to be 0.81 . Also, by using the Cronbach's Alpha method, the coefficients' validity for self management, competence, and communication components were obtained as equal to $0.83,0.67$, and 0.81 , respectively.

\section{Results}

A total of 235 elderly persons aged between 60 to 75 years and residing in the city of Tehran participated in this study. There were 100 women and 135 men. Regarding their educational level, 124 had studied below the level of high school diploma, 57 held high school diplomas, and 54 had university education.

With the help of correlation matrix to examine the connection between the research variables, the results from data analysis showed that there was a significant and positive correlation between self management variables and mental vitality $(\mathrm{r}=0.69, \mathrm{P}<0.01)$. There was also a positive and significant correlation between competence variables and mental vitality $(\mathrm{r}=0.53, \mathrm{P}<0.01)$. In addition, there was a significant and positive connection between communication variables and mental vitality $(\mathrm{r}=0.58, \mathrm{P}<0.01)$.

To identify which of the self management, competence, and communication variables played a greater role in anticipating mental vitality in the elderly, the Stepwise Regression Analysis was employed. Results of this investigation are reported in Table 1.

Considering the results shown in Table 1, fulfilling the need for self management anticipated the mental vitality in the elderly in a positive and significant way $(\mathrm{P}<0.001$ and $\beta=0.48)$. This variable alone explained $23 \%$ of the variance of mental vitality of the elderly. In the second step, the need for self management and communication $(\mathrm{P}<0.001$ and $\beta=0.26)$ anticipated mental vitality in a significant way, and by adding the need for communication to the equation, $6 \%$ was added to the coefficient of determination $\left(\mathrm{R}^{2}=0.29\right)$. In the third step, mental vitality was significantly anticipated by all the three needs of self management, communication, and competence $(\beta=0.14)$ and 0.01 ). By the entry of the need for communication to

Table 1. Results of stepwise regression for anticipating mental vitality based on basic psychological needs

\begin{tabular}{|c|c|c|c|c|c|c|c|}
\hline Indica & $\mathbf{F}$ & $\mathbf{P}$ & Beta & $\mathbf{T}$ & $\mathbf{P}$ & $\mathbf{R}$ & $\mathbf{R}^{2}$ \\
\hline Self management & 70.19 & 0.001 & 0.48 & 8.37 & 0.001 & 0.48 & 0.23 \\
\hline $\begin{array}{l}\text { Self management and } \\
\text { communication }\end{array}$ & 48.81 & 0.001 & 0.26 & 4.61 & 0.001 & 0.54 & 0.29 \\
\hline $\begin{array}{l}\text { Self-management, } \\
\text { communication, and } \\
\text { competence }\end{array}$ & 35.58 & 0.001 & 0.14 & 2.59 & 0.01 & 0.56 & 0.31 \\
\hline
\end{tabular}


the equation, $2 \%$ was added to the coefficient of determination $\left(\mathrm{R}^{2}=0.31\right)$.

\section{Conclusion}

Results of the study showed that from among the basic psychological needs, the need for self management, communication, and competence had the greatest share in anticipating mental vitality in the elderly. To sum it all up, according to the self determination theory [4], these three needs are the foundation of one's behavior. Therefore, satisfying these three needs leads to better growth, health and mental vitality in the elderly. Accordingly, recognizing the basic psychological needs and paying attention to them can be an effective solution for preserving and increasing the mental vitality of the elderly people.

\section{Acknowledgements}

This research did not receive any specific grant from funding agencies in the public, commercial, or not-forprofit sectors.

\section{Conflict of Interest}

The authors declared no conflicts of interest. 


\title{
رابطه نيازهاى اساسى روانشناختى با سرزندگى ذهنى در سالمندان
}

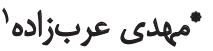

1- كروه روانشئاسى تربيتى، دانشكده روانشاسىى وعلوم تربيتى، دانشكاه خوارزمى، تيران، ايران.

\begin{abstract}
בת

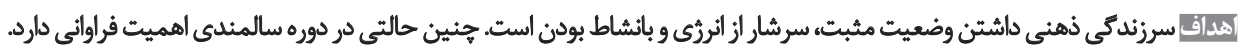

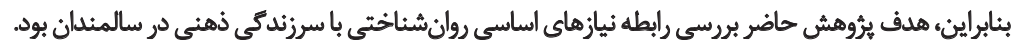

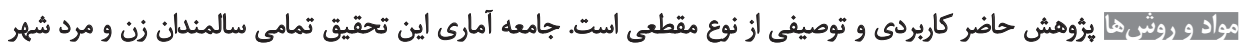

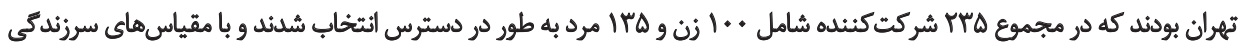

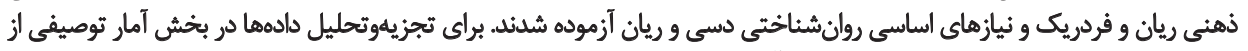

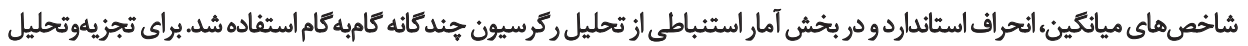

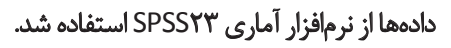

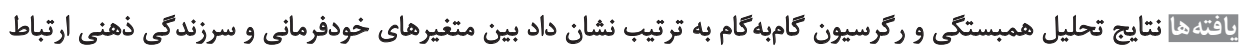

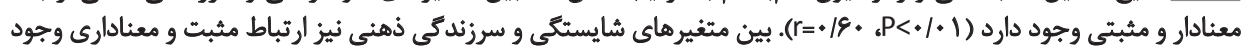

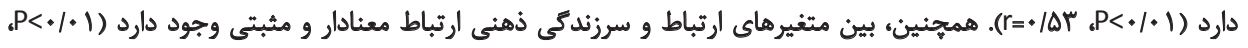

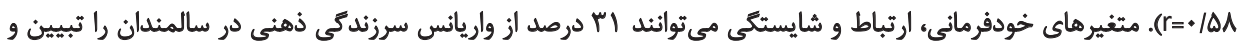
بيشىبينى كنيند.

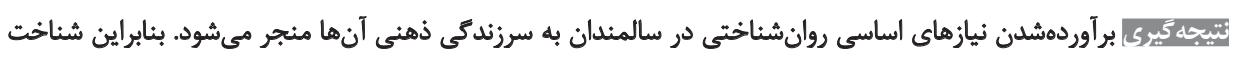

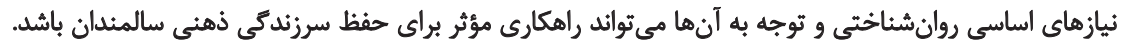

تاريخ دريافت: با دى هوسا

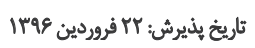

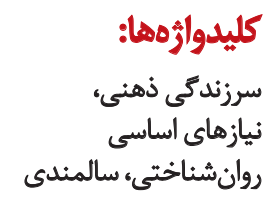

كليدوازٔهها:

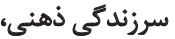

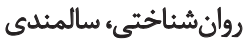

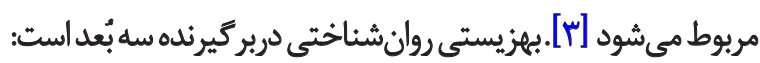

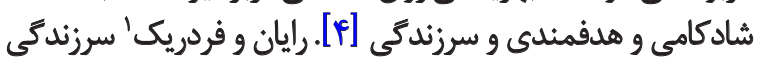

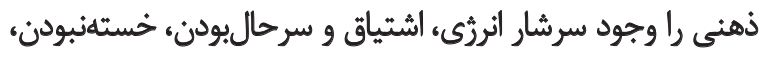

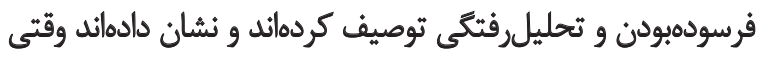

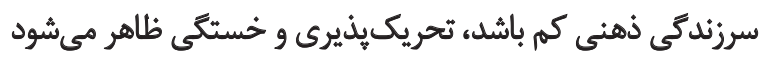

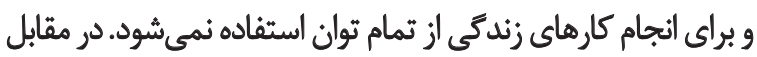

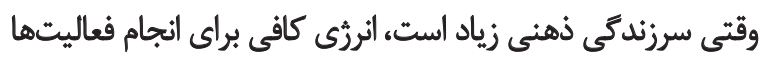

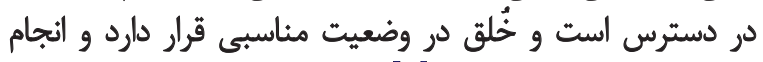

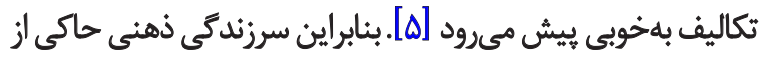

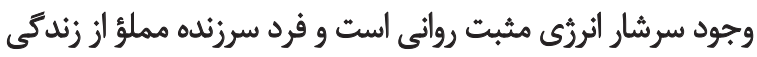

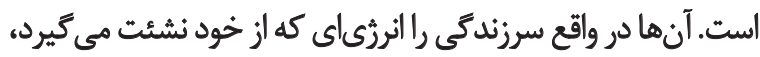

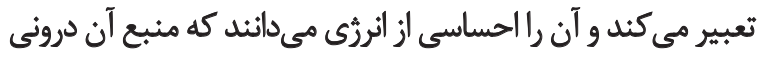

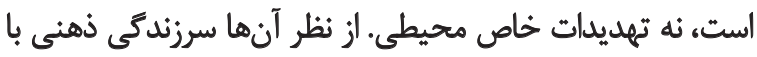

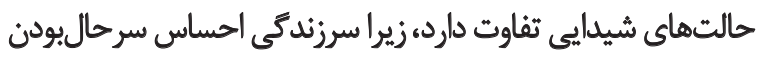

يكى از مسائل عمده جمعيتى در كشورهاى جهان مسئله

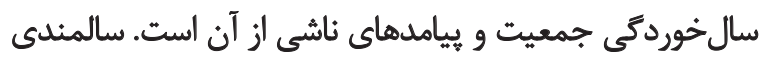

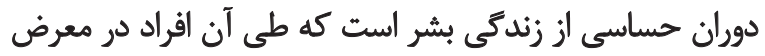

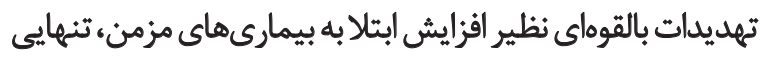

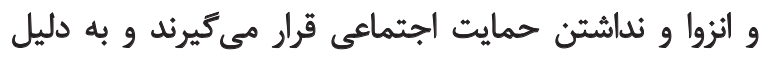

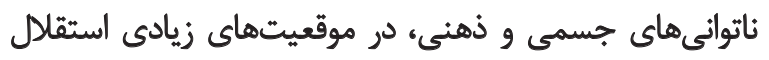

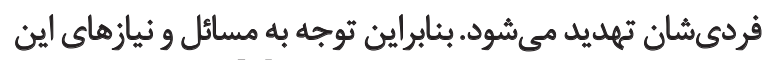

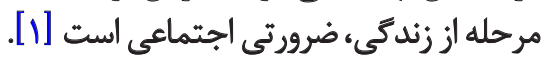

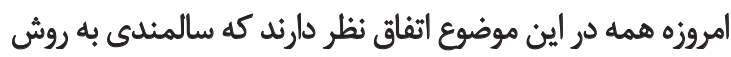

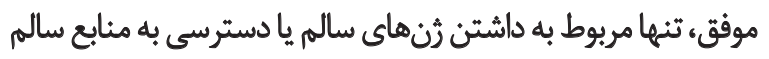

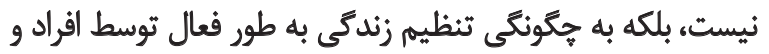

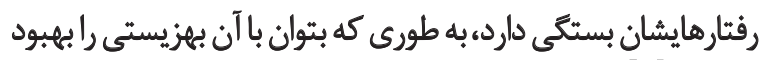

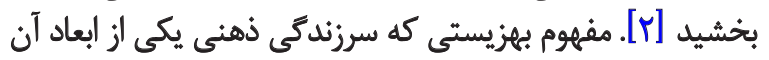

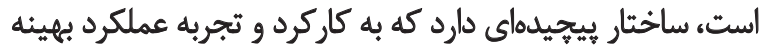

\section{드.}

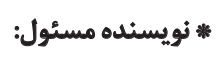

دكتر مهدى عربزاده

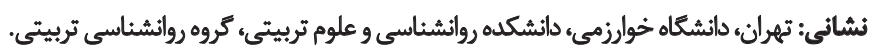

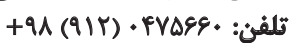

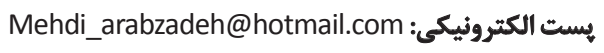


ذهنى دائشآموزان به كمك نيازهاى اساسى روانشناختى و

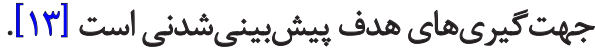
اكرجه بيشتر اين تحقيقات نشان مىدهد كه برآوردهشن

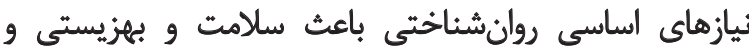

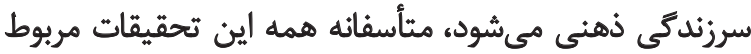

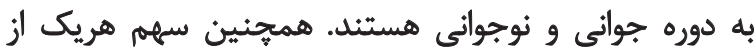

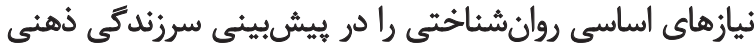

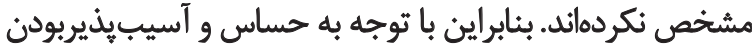

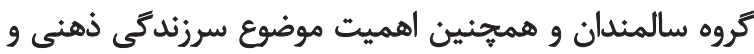

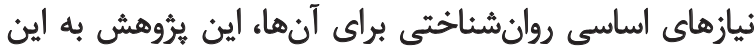

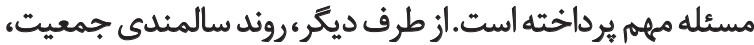

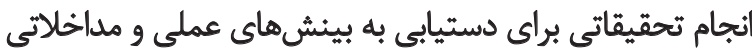

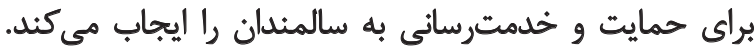

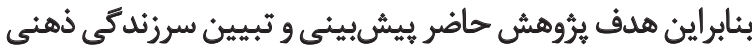

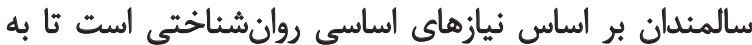

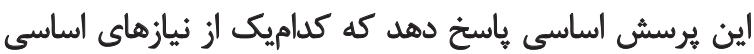

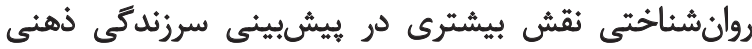

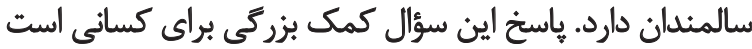
كه به نوعى در حوزه سالمندى فعاليت دارئد.

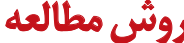

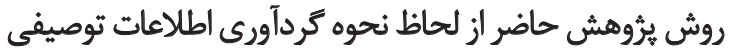

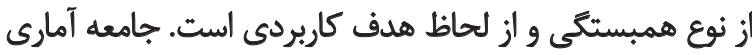

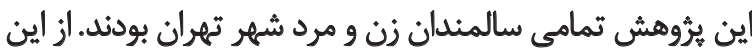

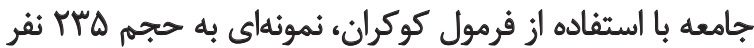

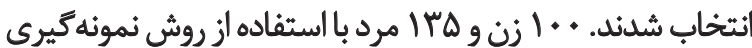

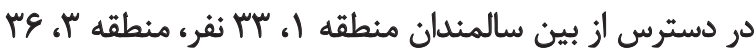

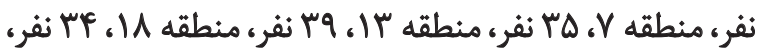

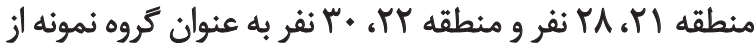

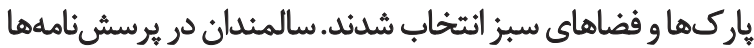

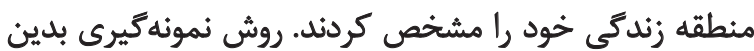

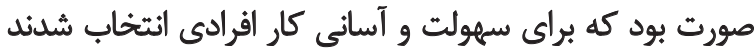

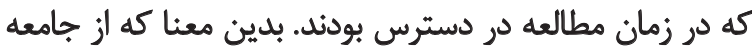

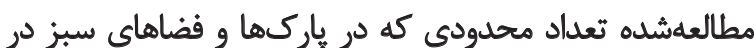

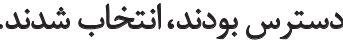

$$
S=\frac{t^{2} \frac{p(1-p)}{d^{2}}}{1+\frac{1}{N}\left[t^{2} \frac{p(1-p)}{d^{2}}-1\right]}
$$

اعضاى نمونه با توجه به معيارهاي ورود و خروج انتخاب شده

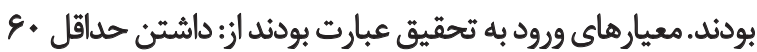

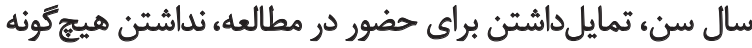
نقص عضو جلى و نداشتن بيمارىهاى حاد حاد جسمى و ورانى.
و يرائرزى بودن است، نه مجبوربودن و هدايتشدن. بنابراين سرزندكى

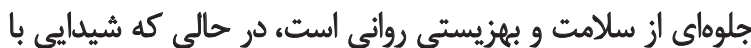

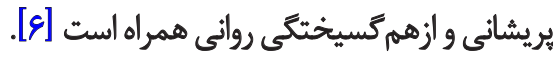
شواهد موجود حاكى از اين است كه سالمندان از نظر تجربه

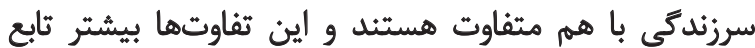

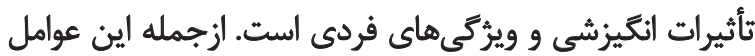

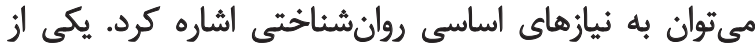

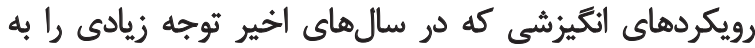

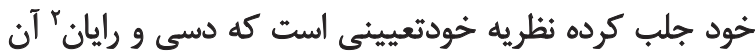

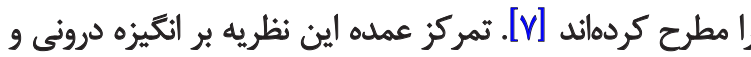

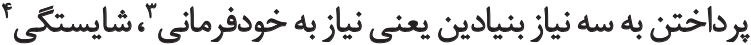

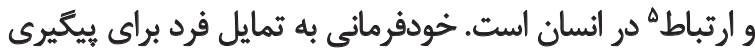

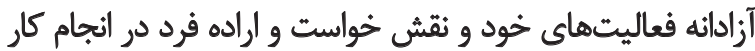

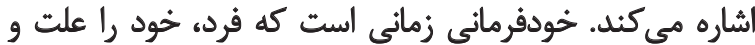

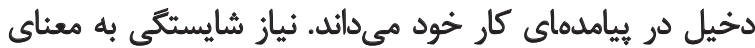

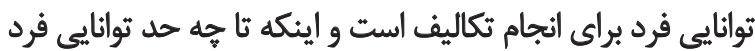

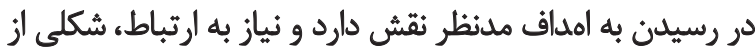

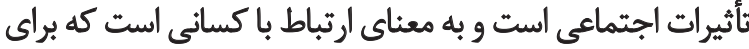

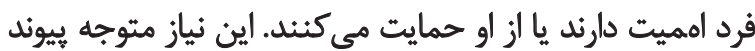

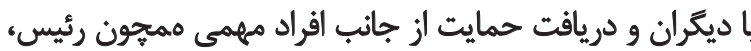
والدين، مراقبان و دوستان و ممكاران است [لم]ـ.

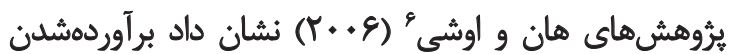

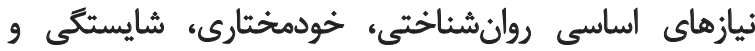
ارتباط به ترتيب همبستكي بالايي با عواطف مثبت ديت در جواناني

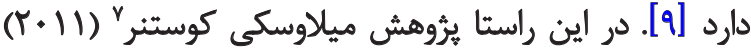

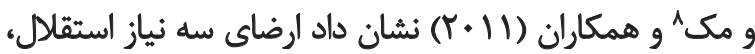

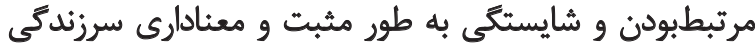

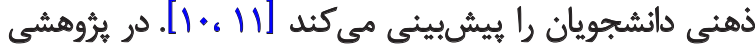

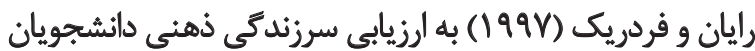

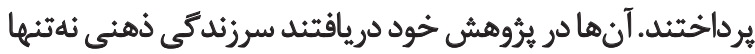

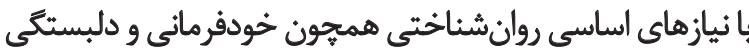

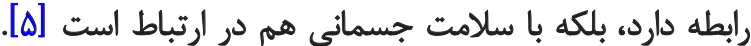

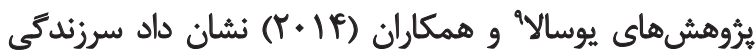

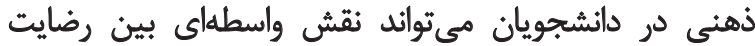

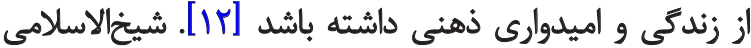

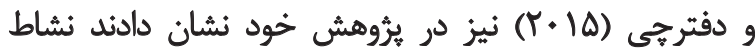

2. Deci \& Ryan

3. Autonomy

4. Competence

5. Relatedness

6. Hahn \& Oish

7. Milyavskaya \& Koestner

8. Mack

9. Uysala 
مقياس با استفاده از روش آلفاى كرائباخ |A|• به دست آمدائ

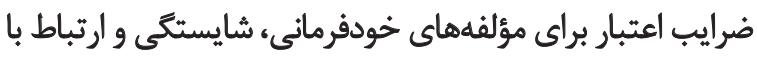

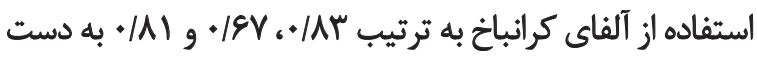

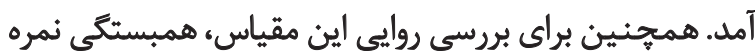

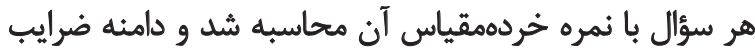

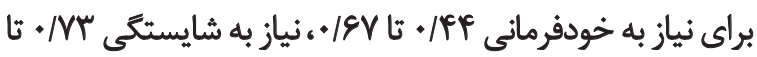

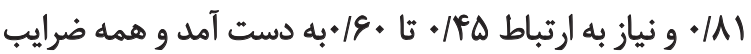

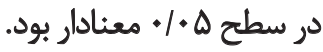

يافتهها

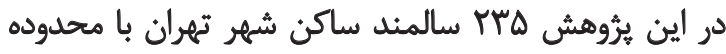

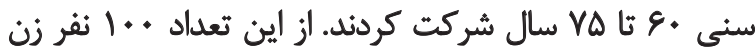

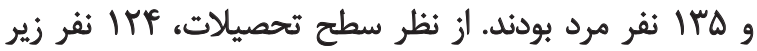

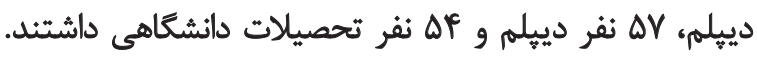

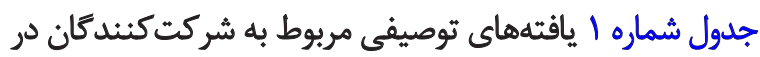

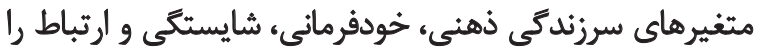

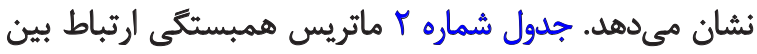

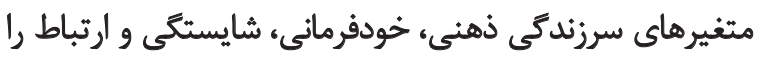

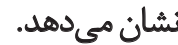

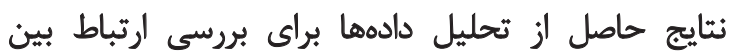

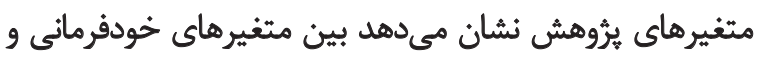

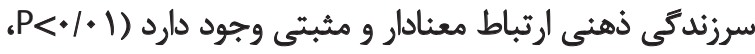

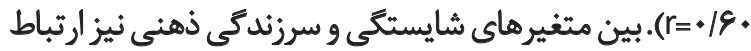

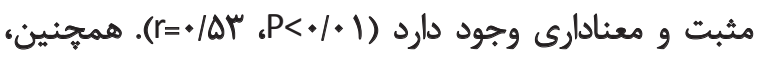

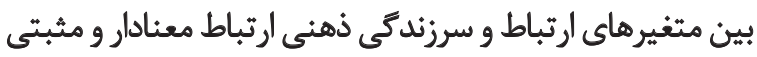

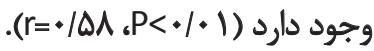

براى مشخصكردن اينكه كداميك از متغيرهاى خودمختارى،

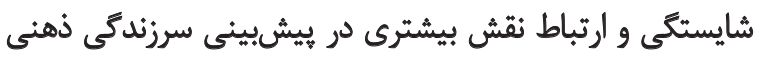

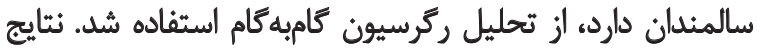

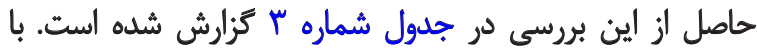

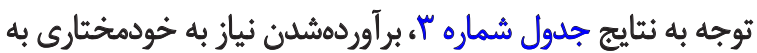

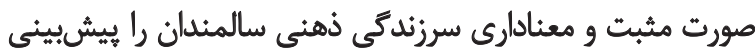

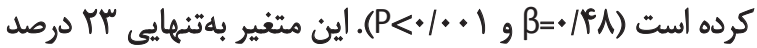

شرايط خروج از تحقيق نيز عبارت بودند از: تمايلنداشتن به ادامه شركت در مطالعه و داشتن بيمارى هائ جسمانى و ورائى حاد. براي تجزيهوتحليل دادهها بر بخش آمار توصيفى از شاخص آناى

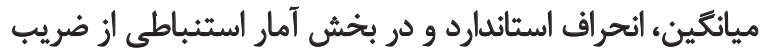

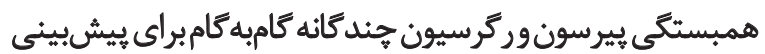

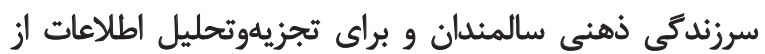

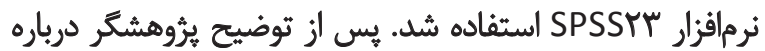

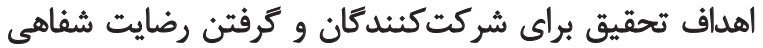

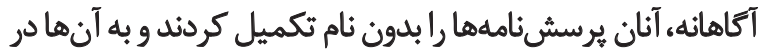

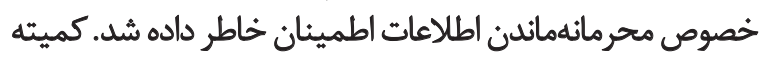

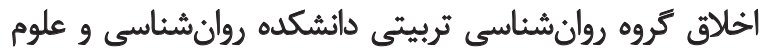

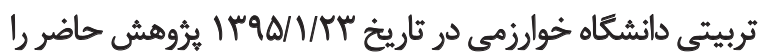

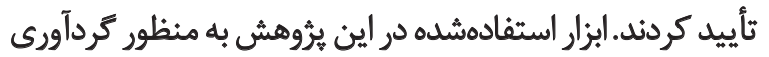

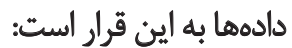

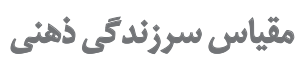

رايان و فردريك بر اساس نظريه خودتعيينى دسى و رايان

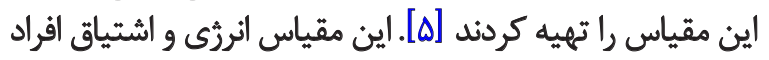

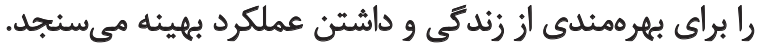

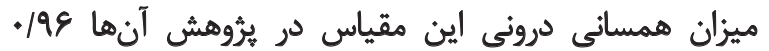

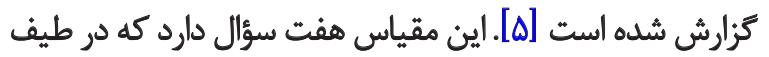

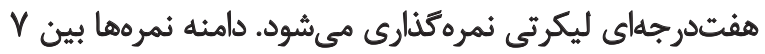

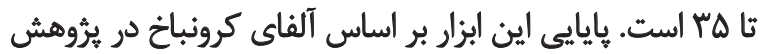

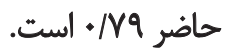

\section{مقياس نيازهاى اساسى روان شناختى}

براى اندازميرى نيازهاى اساسى روانشناختى از مقياس

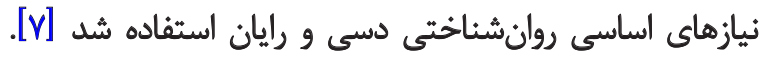

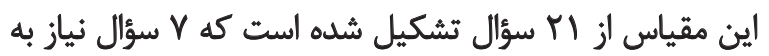

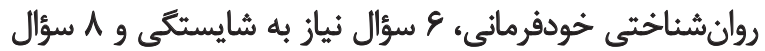

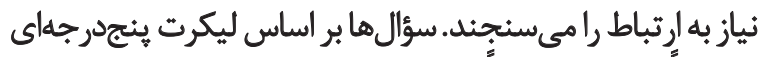

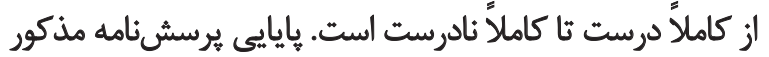

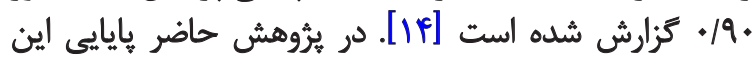

جدول ا. يافتهاى توصيفى مربوط به شركت كنيدكان در متغيرهاى يُوهش

\begin{tabular}{|c|c|c|c|}
\hline تعداد & انحراف استاندارد & مياتكين & متغيرها \\
\hline Mra & $H / \Delta S$ & $r \cdot / \mu r$ & سرزندكى ذهنى \\
\hline ma & $g / M F$ & WNT & خودفرمانى \\
\hline D & $\Delta / \vartheta$ & IE/eV & شايستكى \\
\hline MTd & e/pa & TV/MI & ارتباط \\
\hline
\end{tabular}

ill 
جدول r. ماتريس همبستكى ارتباط بين متغيرهاي ثئزوهش

\begin{tabular}{|c|c|c|c|c|}
\hline$f$ & $r$ & $r$ & 1 & متغير \\
\hline & & & 1 & سرزئدىى ذهنى \\
\hline & & 1 & $.18 * *$ & خودفُرمانى \\
\hline & 1 & . $p v^{* 0}$ &.$/ \Delta T^{\prime}$ & شايستكى \\
\hline 1 &.$|\Delta|^{* *}$ &.$/ 19 q^{* *}$ &.$/ \mathrm{AM}^{* *}$ & ارثباط \\
\hline
\end{tabular}

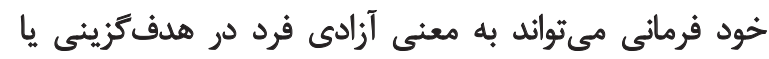

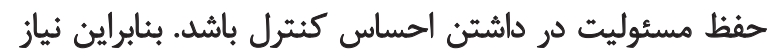

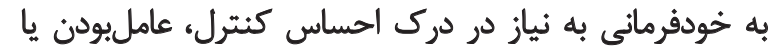

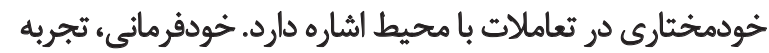

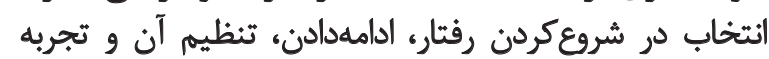

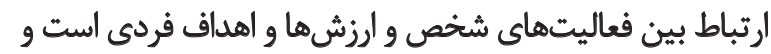

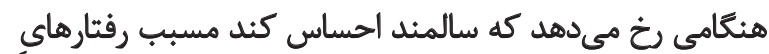

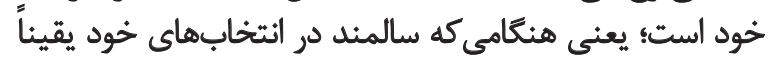

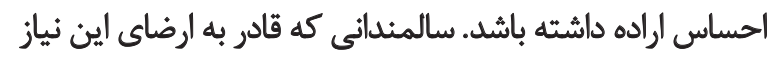

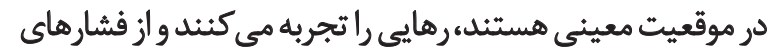

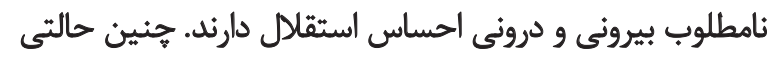

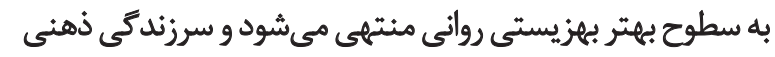

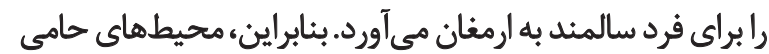

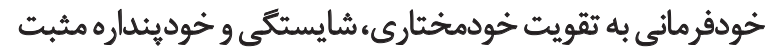

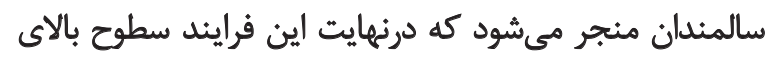

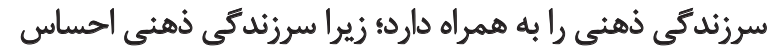

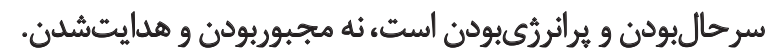

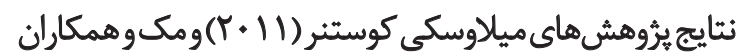

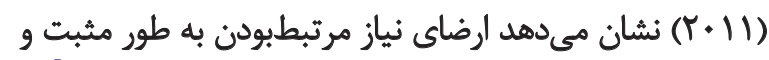

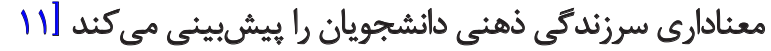

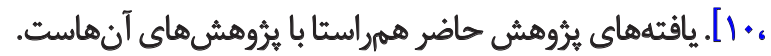

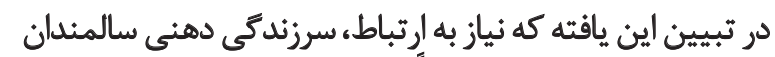

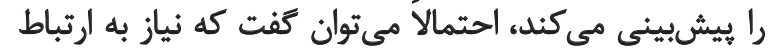

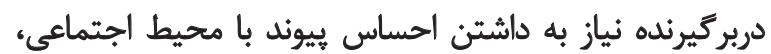

از واريانس سرزندكى ذهنى سالمندان را تبيين كرده است. در كام بام

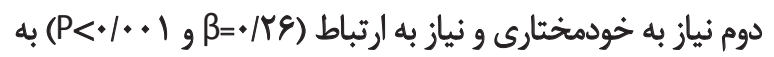

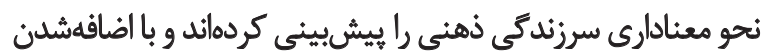

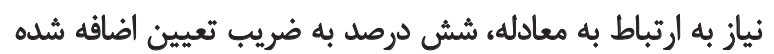

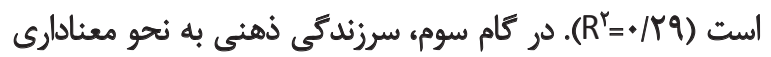

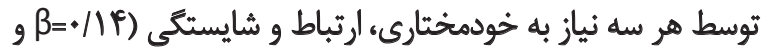

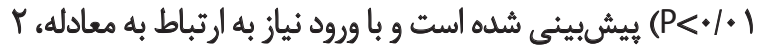

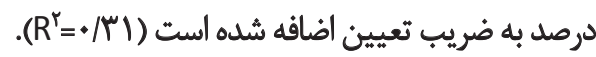

ب بحث

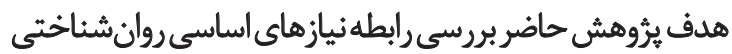

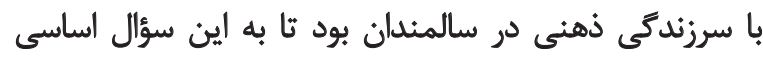

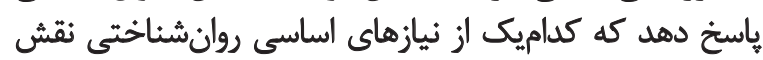

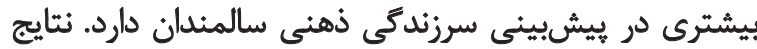

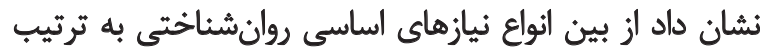

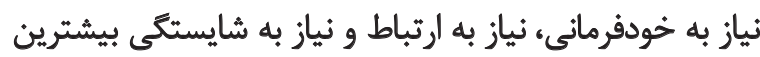

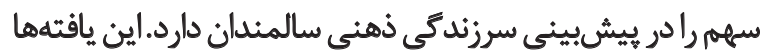

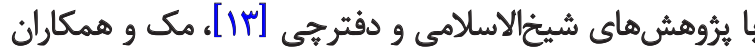

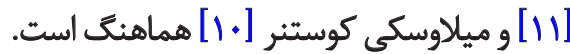

براى مثال، نتايج تحقيق رايان و فردريك (199V) نشان

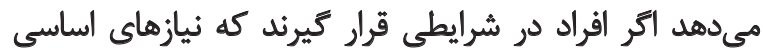

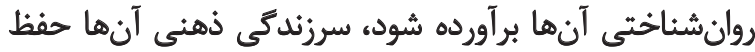

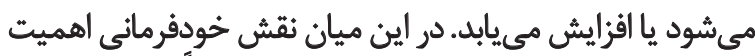

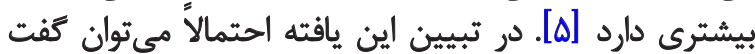

جدول ب. نتايج رترسيون كامبه كام براى بيشبينى سرزندّى ذهنى بر اساس نيازهاى اساسى روانشئاختى

\begin{tabular}{|c|c|c|c|c|c|c|c|}
\hline $\mathrm{R}^{2}$ & $\mathbf{R}$ & $\mathbf{P}$ & $\mathrm{T}$ & Beta & $\mathbf{P}$ & $F$ & شاخصر \\
\hline . & $\cdot / 4 \wedge$ & $+1+.1$ & NHY & $\cdot / \uparrow \wedge$ & $.1+.1$ & $6 \cdot / 19$ & خودفرمائى \\
\hline.$/ 7 q$ &.$/ \Delta t^{5}$ & $.1 . .1$ & $p(8)$ &.$/ 48$ & $.1 . .1$ & PNAI & خودفرمانى و ارتباط \\
\hline.$/ m 1$ & .108 & .1 .1 & $r / \Delta q$ &.$/ F$ &.$/ . .1$ & $\mathrm{r} \Delta / \mathrm{AA}$ & خودفرمائي، ارتباط و شايستكى \\
\hline
\end{tabular}




$$
\text { تشكر و قدردانى }
$$

از تمامى سالمندان شريف شهر تهران به دليل شركت در اين

تحقيق تشكر و قدردانى مى شود. مقاله حامى مالى نداشئه بـ است

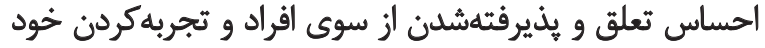

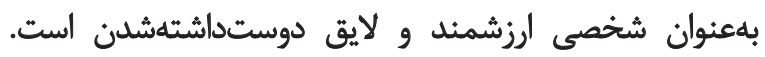

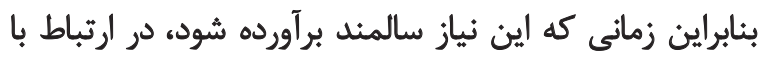

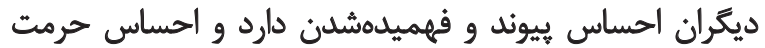

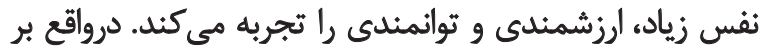

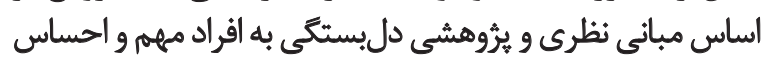

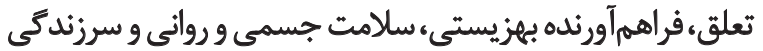

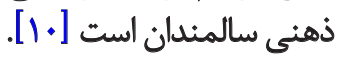

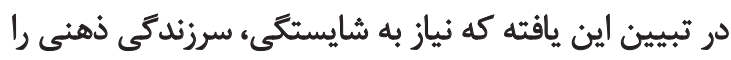

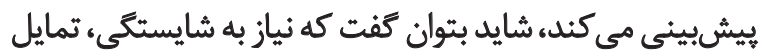

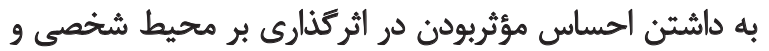

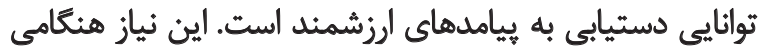

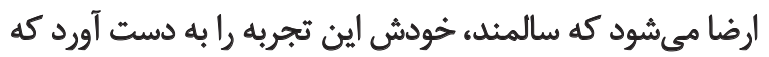

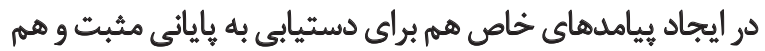

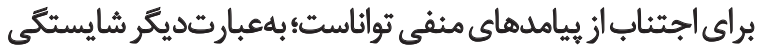

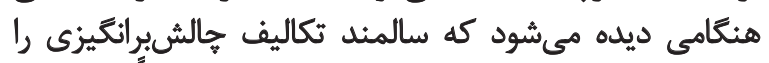

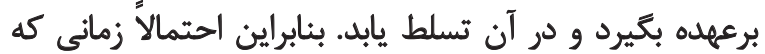

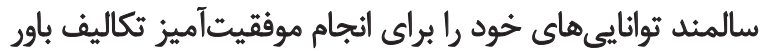

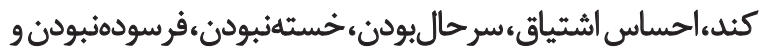

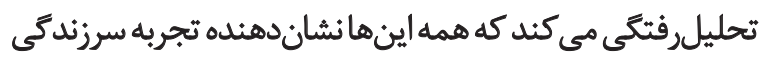
ذهنى زياد است.

درمجموع، طبق نظريه خودتعيينى [f]، اين سه نياز زيربناي

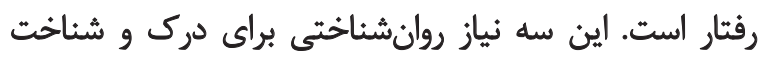

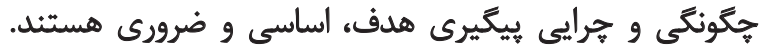

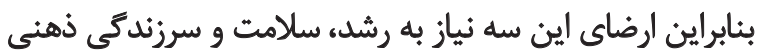

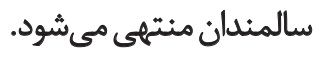

مهمترين محدويت يُروهش حاضر استفاده از نمونه در

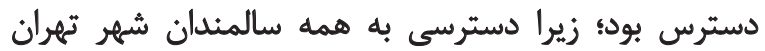

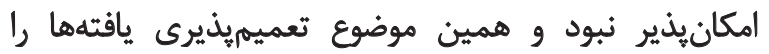

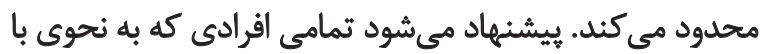

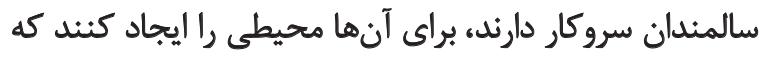

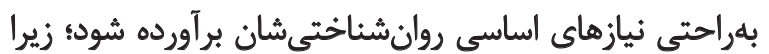

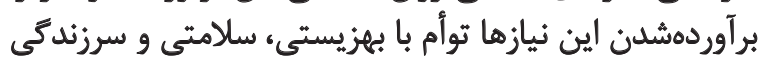

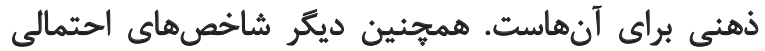
مؤثر در سرزندگى ذهنى سالمندان را بررسى كنند.

\section{نتيجهيَيرىنهايى}

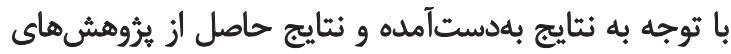

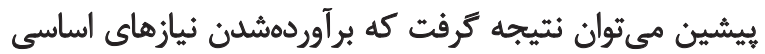

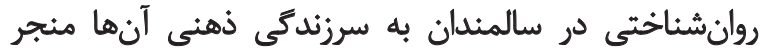

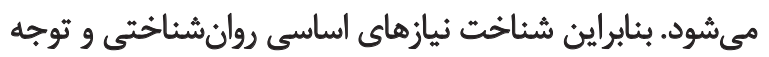

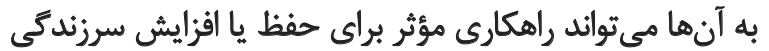

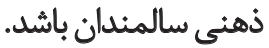




\section{References}

[1] Darvishpoor Kakhki A, Abed Saeedi J, Delavar A, Saeed-OZakerin M. [Tools for measurement of health status and quality of life of elderly people (Persian)]. Research in Medicine. 2010; 33(3):162-171.

[2] Pachana N, Laidlaw K. The Oxford handbook of clinical geropsychology. Oxford: Oxford University Press; 2014.

[3] Deci EL, Ryan RM. The "What" and "Why" of goal pursuits: Human needs and the self-determination of behavior. Psychological Inquiry. 2000; 11(4):227-68. doi: 10.1207/s15327965pli1104_01

[4] Ryan RM, Deci EL. Intrinsic and extrinsic motivations: Classic definitions and new directions. Contemporary Educational Psychology. 2000; 25(1):54-67. doi: 1006/ceps.1999.1020

[5] Ryan RM, Frederick C. On energy, personality, and health: Subjective vitality as a dynamic reflection of well-being. Journal of Personality. 1997; 65(3):529-65. doi: 10.1111/j.1467-6494.1997. tb00326.x

[6] Ryan RM, Deci EL. From ego depletion to vitality: Theory and findings concerning the facilitation of energy available to the self. Social and Personality Psychology Compass. 2008; 2(2):702-17. doi: 10.1111/j.1751-9004.2008.00098.x

[7] Deci EL, Ryan RM. The handbook of self-determination research. Rochester: University of Rochester Press; 2002

[8] Janssen S, van Vuuren M, de Jong MDT. Identifying support functions in developmental relationships: A self-determination perspective. Journal of Vocational Behavior. 2013; 82(1):20-9. doi: 10.1016/j.jvb.2012.09.005

[9] Hahn J, Oishi S. Psychological needs and emotional well-being in older and younger Koreans and Americans. Personality and Individual Differences. 2006; 40(4):689-98. doi: 10.1016/j. paid.2005.09.001

[10] Milyavskaya M, Koestner R. Psychological needs, motivation, and well-being: A test of self-determination theory across multiple domains. Personality and Individual Differences. 2011; 50(3):387-91. doi: 10.1016/j.paid.2010.10.029

[11] Mack DE, Wilson PM, Oster KG, Kowalski KC, Crocker PRE, Sylvester BD. Well-being in volleyball players: Examining the contributions of independent and balanced psychological need satisfaction. Psychology of Sport and Exercise. 2011; 12(5):533-9. doi: 10.1016/j.psychsport.2011.05.006

[12] Uysala R, Satici SA, Satici B, Akin A. Subjective vitality as mediator and moderator of the relationship between life satisfaction and subjective happiness. Educational Sciences: Theory Practice. 2014; 14(2):489-497. doi: 10.12738/estp.2014.2.1828

[13] Sheikholeslami R, Daftarchi E. [The prediction of students' subjective vitality by goal orientations and basic psychological needs (Persian)]. Journal of Psychology. 2015; 19(2):147-174.

[14] Wei M, Shaffer PA, Young SK, Zakalik RA. Adult attachment, shame, depression, and loneliness: The mediation role of basic psychological needs satisfaction. Journal of Counseling Psychology. 2005; 52(4):591-601. doi: 10.1037/0022-0167.52.4.591 
